\title{
Mechanisms of magnoliae cortex on treating sarcopenia explored by GEO gene sequencing data combined with network pharmacology and molecular docking
}

\author{
Xingqi Zhao ${ }^{1 \dagger}$, Feifei Yuan ${ }^{2 \dagger}$, Haoyang Wan ${ }^{1}$, Hanjun Qin ${ }^{1}$, Nan Jiang ${ }^{1 *}$ (D) and Bin Yu ${ }^{1 *}$ (D)
}

\begin{abstract}
Background: Administration of Magnoliae Cortex (MC) could induce remission of cisplatin-induced sarcopenia in mice, however, whether it is effective on sarcopenia patients and the underlying mechanisms remain unclear.

Methods: Sarcopenia related differentially expressed genes were analysed based on three Gene Expression Omnibus (GEO) transcriptome profiling datasets, which was merged and de duplicated with disease databases to obtain sarcopenia related pathogenic genes. Gene ontology (GO) and Kyoto Encyclopedia of Genes and Genomes (KEGG) analysis were than performed to analyse the role of proteins encoded by sarcopenia related pathogenic genes and the signal regulatory pathways involved in. The main active components and target proteins of $M C$ were obtained by searching traditional Chinese medicine network databases (TCMSP and BATMAN-TCM). MC and sarcopenia related pathogenic genes shared target proteins were identified by matching the two. A protein-protein interaction network was constructed subsequently, and the core proteins were filtered according to the topological structure. GO and KEGG analysis were performed again to analyse the key target proteins and pathways of $\mathrm{MC}$ in the treatment of sarcopenia, and build the herbs-components-targets network, as well as core targets-signal pathways network. Molecular docking technology was used to verify the main compounds-targets.
\end{abstract}

Results: Sarcopenia related gene products primarily involve in aging and inflammation related signal pathways. Seven main active components (Anonaine, Eucalyptol, Neohesperidin, Obovatol, Honokiol, Magnolol, and betaEudesmol) and 26 target proteins of MC-sarcopenia, of which 4 were core proteins (AKT1, EGFR, INS, and PIK3CA), were identified. The therapeutic effect of MC on sarcopenia may associate with PI3K-Akt signaling pathway, EGFR tyrosine kinase inhibitor resistance, longevity regulating pathway, and other cellular and innate immune signaling pathways.

Conclusion: MC contains potential anti-sarcopenia active compounds. These compounds play a role by regulating the proteins implicated in regulating aging and inflammation related signaling pathways, which are crucial in

\footnotetext{
*Correspondence: hnxyjn@smu.edu.cn; yubin@smu.edu.cn ${ }^{+}$Xingqi Zhao and Feifei Yuan contributed equally to this work.

${ }^{1}$ Division of Orthopaedics and Traumatology, Department of Orthopaedics \& Guangdong Provincial Key Laboratory of Bone and Cartilage Regenerative Medicine, Nanfang Hospital, Southern Medical University, Guangzhou 510515, China

Full list of author information is available at the end of the article
} original author(s) and the source, provide a link to the Creative Commons licence, and indicate if changes were made. The images or other third party material in this article are included in the article's Creative Commons licence, unless indicated otherwise in a credit line to the material. If material is not included in the article's Creative Commons licence and your intended use is not permitted by statutory regulation or exceeds the permitted use, you will need to obtain permission directly from the copyright holder. To view a copy of this licence, visit http://creativecommons.org/licenses/by/4.0/. The Creative Commons Public Domain Dedication waiver (http://creativeco mmons.org/publicdomain/zero/1.0/) applies to the data made available in this article, unless otherwise stated in a credit line to the data. 
pathogenesis of sarcopenia. Our study provides new insights into the development of a natural therapy for the prevention and treatment of sarcopenia.

Keywords: Sarcopenia, Magnoliae Cortex, Network pharmacology, Compound-target relationship, Gene ontology, KEGG, Molecular docking

\section{Background}

Sarcopenia is a progressive skeletal muscle disorder, characterized by low muscle strength, low muscle quantity/ quality, as well as low physical performance according to the level of disease progression [1]. With the progression of sarcopenia, the incidence of adverse outcomes increases gradually, such as fractures $[2,3]$, physical disability [4], and mortality [5]. However, at present, there are limited preventive and therapeutic interventions for this disease [6].Therefore, new therapies for sarcopenia are urgently needed to intervene or delay adverse health outcomes.

One of the reasons why sarcopenia lacks effective treatment measures is that the pathogenesis is not fully understood, thus lack of intervention targets. Considering loss of muscle strength and mass is also a fundamental feature of aging, results of preclinical and clinical studies comparing young and aged individuals suggested that chronic low-grade inflammation contribute to a loss of muscle plasticity during aging [7]. It has been shown that NF-kB signaling and inflammatory cytokines also take part in the creation and maintenance of sarcopenia status [8]. Therefore, we speculated interventions against aging and inflammation may benefit sarcopenia. A recent in vivo study demonstrated that Magnoliae Cortex (MC), an herbal medicine widely used in medical practice of traditional Chinese medicine (TCM), could alleviate cisplatin-induced sarcopenia [9]. This result reminds us that MC might be a new drug to intervene and delay adverse consequences of sarcopenia.

As the wealth of China and the world, TCM has attracted more and more attention in the prevention and treatment of a series of diseases for the advantages of definite curative effect, safety, and few side effects. Different from the single targeted therapy of Western Medicine, herbal medicine of TCM mainly carries out multi-target treatment because they contain a large number of active chemical components. MC is called Houpu in Chinese herb (scientific term: Magnolia Officinalis Rehd Et Wils.), belongs to dampness removing drugs in TCM theory [10]. Recent pharmacological analysis have pointed out that $\mathrm{MC}$ has the effects of anxiolytic-like [11], apoptosisinducing and antitumor [12], antimicrobial against multi-drug resistant Staphylococcus aureus [13], as well as lipid metabolism regulation [14]. Although previous studies have shown that $\mathrm{MC}$ can alleviate cisplatin-induced sarcopenia through immune regulation and inhibiting the expression of inflammatory cytokines [9], the specific active components, cellular and molecular mechanisms remain unclear. There are few or no systematic researches on the biological basis of TCM herbal medicine for treating sarcopenia. How to develop new methods to detect the key components for treating sarcopenia and speculate the possible mechanism not only provides the benefit therapy strategy for the precise treatment of sarcopenia, but also provides methodological reference for the analysis of the possible mechanisms.

Systems biology [15] and network pharmacology [16, 17] have been successfully applied in the targets prediction and mechanisms research in treatment of diseases with TCM. For example, Yang et al. used network pharmacology to decipher the cellular and molecular mechanisms of 8 different TCM formulas in the treatment of cardiovascular diseases [18]; Wang et al. expounded the molecular mechanism of 3 different TCM formulas in treating rheumatoid arthritis based on network pharmacology-based approach [19], etc. In recent years, system or network pharmacology combined with multi-omics analysis have shown unique advantages in predicting and interpreting the pharmacological principle of TCM herbs and their mechanism of action in treating various diseases [20-23].

Under the premise of preclinical effectiveness in cisplatin-induced sarcopenia model, we wondered whether MC could also alleviate sarcopenia in clinical patients. In this study, we first looked for target genes/proteins that may interfere with the disease process through the sequencing data of sarcopenia muscle biopsies, and combined with the sarcopeniarelated genes databases to obtain sarcopenia related pathogenic genes/proteins. Then, we used the network pharmacology method to predict the targets of MC, and matched them with sarcopenia related pathogenic genes to obtain MC-sarcopenia targets. Afterwards, the mechanism was systematically predicted according to protein functions and involved signal pathways. Finally, molecular docking technology was used to verify whether the active components of MC play a role in sarcopenia related pathogenic proteins. A research flow chart of the method was shown in Fig. 1. 


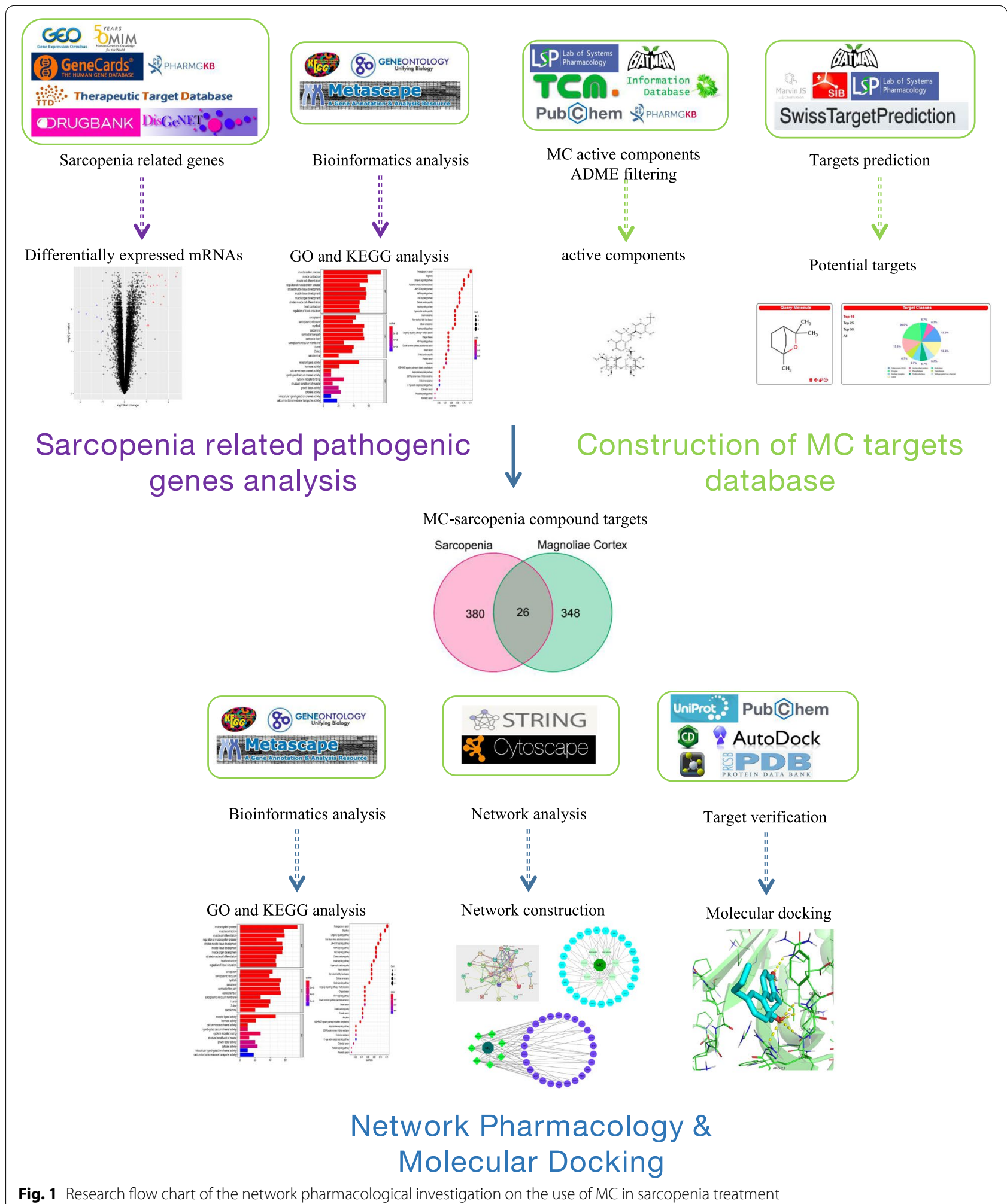




\section{Materials and methods}

\section{Construction of sarcopenia related pathogenic genes} database

First, high-throughput sequencing data of mRNAs in the muscle biopsies of healthy and sarcopenia elderly people was obtained from the Gene Expression Omnibus (GEO) database (https://www.ncbi.nlm.nih.gov/geo/). We chose the following three series for analysis, including GSE111006, GSE111010, and GSE111016, as the individuals they included were the elderly with or without sarcopenia. Sva and Limma of R 3.6.3 were used to carry out data integration of multiple series and correct data batches effect. Genes with an adjusted $P$ value $<0.05$ and absolute value of $\log _{2}$ (Fold Change) $>1$ were considered as significantly differentially expressed and sarcopenia related pathogenic genes. In addition, sarcopenia related pathogenic genes were integrated with the diseaserelated genes database, including GeneCard database (https://www.genecards.org/), OMIM database (https:// www.omim.org/), Pharmgkb (https://www.pharmgkb. org/), TTD database (http://db.idrblab.net/ttd/) [24], DrugBank database (https://go.drugbank.com/) [25], and DisGeNET database (https://www.disgenet.org) [26], using "sarcopenia" as keyword. Subsequently, the duplicated genes were removed, and the sarcopenia related pathogenic genes database was established.

\section{Gene ontology (GO) and Kyoto Encyclopedia of Genes and Genomes (KEGG) analysis}

After obtaining the sarcopenia related pathogenic genes, we used ClusterProfiler [27] of R 3.6.3 to conduct the GO and KEGG analysis [28]. The related software packages can be obtained from https://www.bioconductor.org/. The GO enrichment mainly analyses the biological process (BP), cellular composition (CC), and molecular function (MF) of the genes, and the KEEG enrichment mainly analyses the potential biological pathways involved in these interested genes.

\section{Construction of MC main active components database}

The TCM system pharmacology database and analysis platform (TCMSP, https://tcmspw.com/tcmsp.php) [29], the bioinformatics analysis tool for the molecular mechanism of TCM (BATMAN-TCM, http://bionet. ncpsb.org.cn/batman-tcm/) [30], and the TCM information database (http://bidd.group/TCMID/) [31] were used to identify the active components of MC. The main active components were then filtered according to the optimal toxicokinetic ADME rules [oral bioavailability $(\mathrm{OB}) \geq 30 \%$, drug-like $(\mathrm{DL}) \geq 0.18$ ] [32]. If the component did not meet the filtering criteria, they were included if they were reported as effective against sarcopenia in relevant literatures $[9,33]$. The molecular structure of components was obtained from the PubChem (https:// pubchem.ncbi.nlm.nih.gov/) or Pharmgkb (https://www. pharmgkb.org/).

\section{Construction of active components potential targets database}

The components of herbal medicine perform related biological functions through relevant targets. In addition to obtaining the targets of the main active components of MC directly from the TCMSP and BATMAN-TCM, the Swiss Target Prediction (http://swisstargetprediction.ch/) [34] were also used to predict possible targets of MC.

\section{Construction of the Protein-Protein interaction (PPI) network}

Based on the above analyses, the targets of main active components were matched with the disease-related pathogenic gene products of sarcopenia to obtain the compound targets of MC-sarcopenia. The Venn map was drawn by venn of R 3.6.3 and the PPI network of the targets was obtained by using the String online tool (https:// string-db.org/) [35]. Then, the GO and KEGG analysis were conducted again to obtain the BP, CC, MF, and potential biological pathways of the compound targets.

\section{Construction of an "Herbs-Components-Targets" network of $\mathrm{MC}$}

Based on the PPI network obtained above, the "HerbsComponents-Targets" network (H-C-T network) of MC was constructed using Cytoscape3.8.2 (https://www. cytoscape.org/) [36]. According to the topological characteristics of the network, the follow three parameters were used to obtain the core composite targets of $\mathrm{MC}$ : degree centrality (DC) [37], closeness centrality (CC) [38], and betweenness centrality (BC) [39]. According to literature reports, the targets with higher than two-fold the median value of DC [40], BC and CC were considered as more accurate core targets [41].

\section{Active components-targets docking}

According to the screened core targets, the active components that may bind to the core targets were searched in reverse to obtain the key components of $\mathrm{MC}$, which were then docked with core targets to verify the accuracy of the main components and prediction targets. The candidate key components crystal structure and the core targets protein structure were downloaded from the PubChem and RCSB protein database (https://www. pdb.org/), respectively. The target proteins preferentially select the structure with molecular binding smaller than $3 \AA$. Then the protein was dehydrated, hydrogenated and ligand separated using Pymol 2.5.1 software (https:// pymol.org/2/). The processed biological macromolecular 
protein was then poured into AutoDockTools 1.5.6 to construct the docking grid box [42, 43]. Molecular docking was completed by using Autodock Vina 1.1.2 software [44], and the molecule with the lowest binding energy in the docking conformation was used to observe the binding effect by matching with the original components and intermolecular interactions.

\section{Results}

\section{Sarcopenia related pathogenic genes}

Joint analysis of three series in the GEO database (GSE111006, GSE111010, GSE111016) identified 28 differentially expressed genes related to sarcopenia in old people (Supplementary Table S1), which were used to build a volcano map (Fig. 2A). In addition, we integrated disease-related pathogenic genes in GeneCard, OMIM, Pharmgkb, and DisGeNET databases to eliminate duplicates, resulting in the identification of 406 sarcopeniarelated pathogenic genes (Supplementary Table S2, Fig. 2B).

GO enrichment analysis was conducted for the identified 406 sarcopenia-related pathogenic genes on CC, MF and BP We selected the top 20 functional enrichment processes to draw a bar diagram (Fig. 2C). In terms of molecular function, also called the biochemical activity of gene products, sarcopenia-related pathogenic gene products mainly involve in the activity regulation of ligand, hormone, channel, receptor, cytokine, such as receptor ligand activity (GO:0,048,018), cytokine activity (GO:0,005,125) (Fig. 2C). Also, sarcopenia-related pathogenic gene products take part in the phosphatidylinositol 3-kinase activity (GO:0,035,004) and 1-phosphatidylinositol-3-kinase activity (GO:0,016,303) (Supplementary Table S3). In the biological process, sarcopenia-related pathogenic gene products mainly involves the system process and cell differentiation of muscle, such as muscle system process (GO:0,003,012), muscle cell differentiation (GO:0,042,692), regulation of muscle system process (GO:0,090,257) (Fig. 2C). Also, sarcopenia-related pathogenic gene products participate in the regulation of inflammatory response (GO:0,050,727), inflammatory cell apoptotic process (GO:0,006,925), regulation of protein kinase B signaling (GO:0,051,896) (Supplementary Table S3).

In addition, we identified the primary signaling pathways involved in sarcopenia by KEGG enrichment analysis, and filtered the top 20 pathways related to sarcopenia (adjusted $P<0.05$ ), including longevity regulating pathway (hsa04211), EGFR tyrosine kinase inhibitor resistance (hsa01521), AMPK signaling pathway (hsa04152), Insulin resistance (hsa04931), FoxO signaling pathway (hsa04068), PI3K-Akt signaling pathway (hsa04151), endocrine resistance (hsa01522) among others (Fig. 2D,
Supplementary Table S4). We listed sarcopenia related pathogenic gene products in several main signaling pathways, and found most of them play important role in related pathways (Supplementary Figures S1-S3).

\section{Active components and target prediction of MC}

A total of 184 active components were obtained from TSMSP, BATMAN-TCM, and TCMID, and four main active components were selected according to the filtering criteria of $A D M E(O B \geq 30 \%$ and $D L \geq 0.18)$. However, Honokiol and Magnolol were verified as two major active components in $\mathrm{MC}$ using high pressure liquid chromatography (HPLC, approximately $0.8 \%$ and $2.1 \%$ in $\mathrm{MC}$ respectively), and related literature confirmed that they showed protective effects in an experimental sarcopenia animal model [9]. In addition, beta-Eudesmol is one of the most studied and major bioactive sesquiterpenes, showed therapeutic potential and pharmacological activities in a series of diseases [33]. Therefore, they were also included although they did not meet the ADME criteria. Finally, seven main active components were included (Table 1). Then, 374 MC target proteins were identified by integrating the data obtained from TCMSP, BATMAN-TCM, and Swiss Target Prediction (Probability $>0.05$ ) (Supplementary Table S5). These target proteins of MC were matched with sarcopenia-related pathogenic gene products, resulting in the selection of 26 composite targets of MC and sarcopenia (Fig. 3A, Supplementary Table S6).

\section{H-C-T network of MC-sarcopenia composite targets}

The MC-sarcopenia composite targets identified were input into STRING to remove the unconnected targets, and the PPI network was obtained (Fig. 3B). Then, the $\mathrm{H}-\mathrm{C}-\mathrm{T}$ network of $\mathrm{MC}$ was constructed using Cytoscape 3.8.2 (Fig. 3C), including 34 nodes and 55 edges.

$\mathrm{GO}$ enrichment analysis showed that the active components of $\mathrm{MC}$ involve in affecting the phosphatidylinositol 3-kinase activity (GO:0,035,004), 1-phosphatidylinositol3-kinase activity (GO:0,016,303) of sarcopenia. Proteins affected by $\mathrm{MC}$ active components participate in the regulation of protein kinase B signaling (GO:0,051,896), response to steroid hormone (GO:0,048,545), inflammatory cell apoptotic process (GO:0,006,925), positive regulation of inflammatory response (GO:0,050,729), and regulation of inflammatory response (GO:0,050,727) as well (Fig. 3D, Supplementary Table S7).

KEGG enrichment analysis showed that proteins affected by $\mathrm{MC}$ active components mainly participate in endocrine resistance (hsa01522), FoxO signaling pathway (hsa04068), PI3K-Akt signaling pathway (hsa04151), EGFR tyrosine kinase inhibitor resistance (hsa01521), and longevity regulating pathway(hsa04211), etc. (Fig. 3E, 


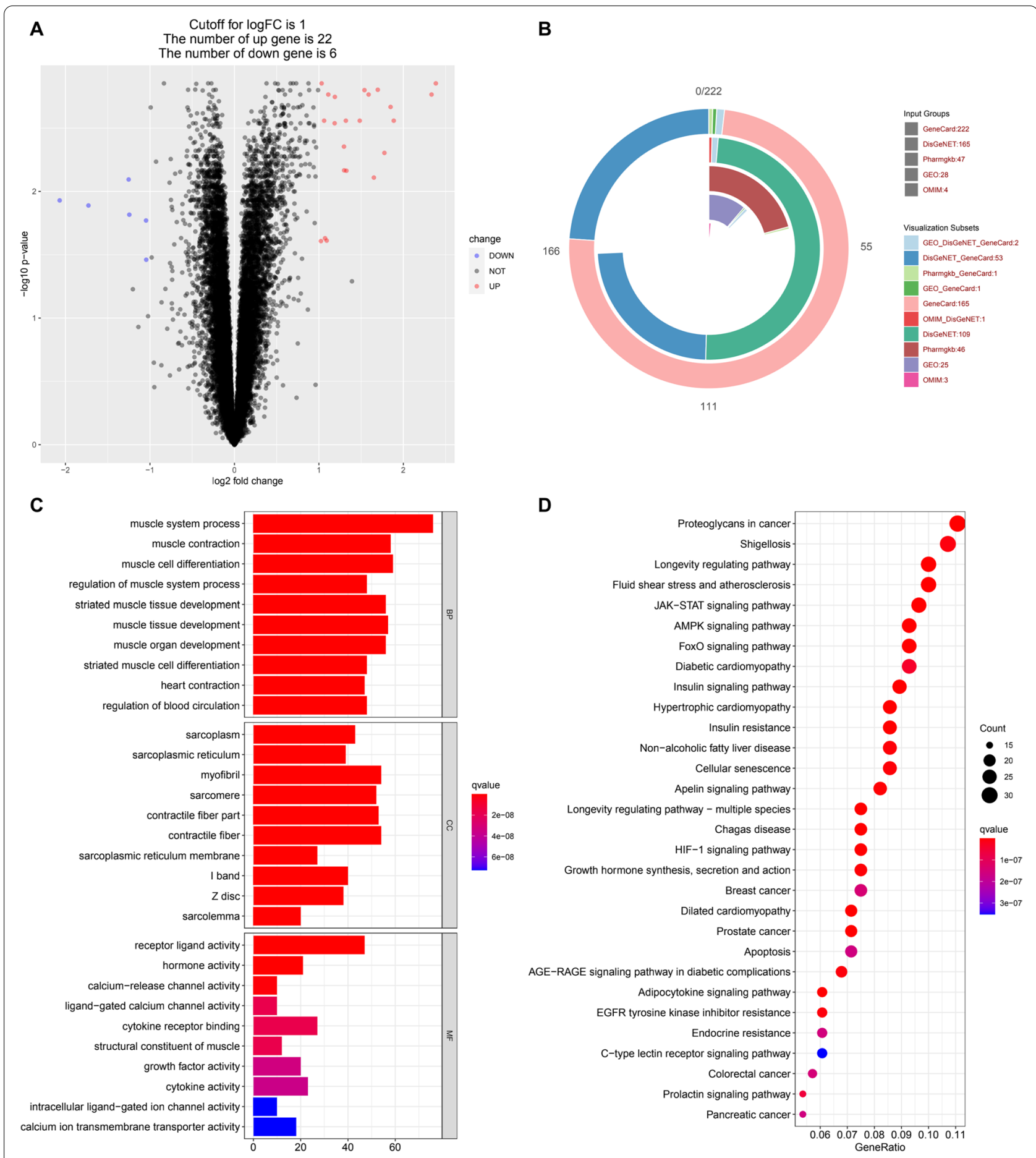

Fig. 2 Sarcopenia related pathogenic genes. A Differential genes volcano map jointly analysed by three GEO series. The fold change of muscle biopsies mRNA in sarcopenia group compared with control group. B Integrated disease-related pathogenic genes in GeneCard, OMIM, Pharmgkb, DisGeNET, and GEO series. C The GO enrichment analysis of sarcopenia related pathogenic gene products. D The KEGG enrichment analysis of sarcopenia related pathogenic gene products 
Table 1 Main components of MC

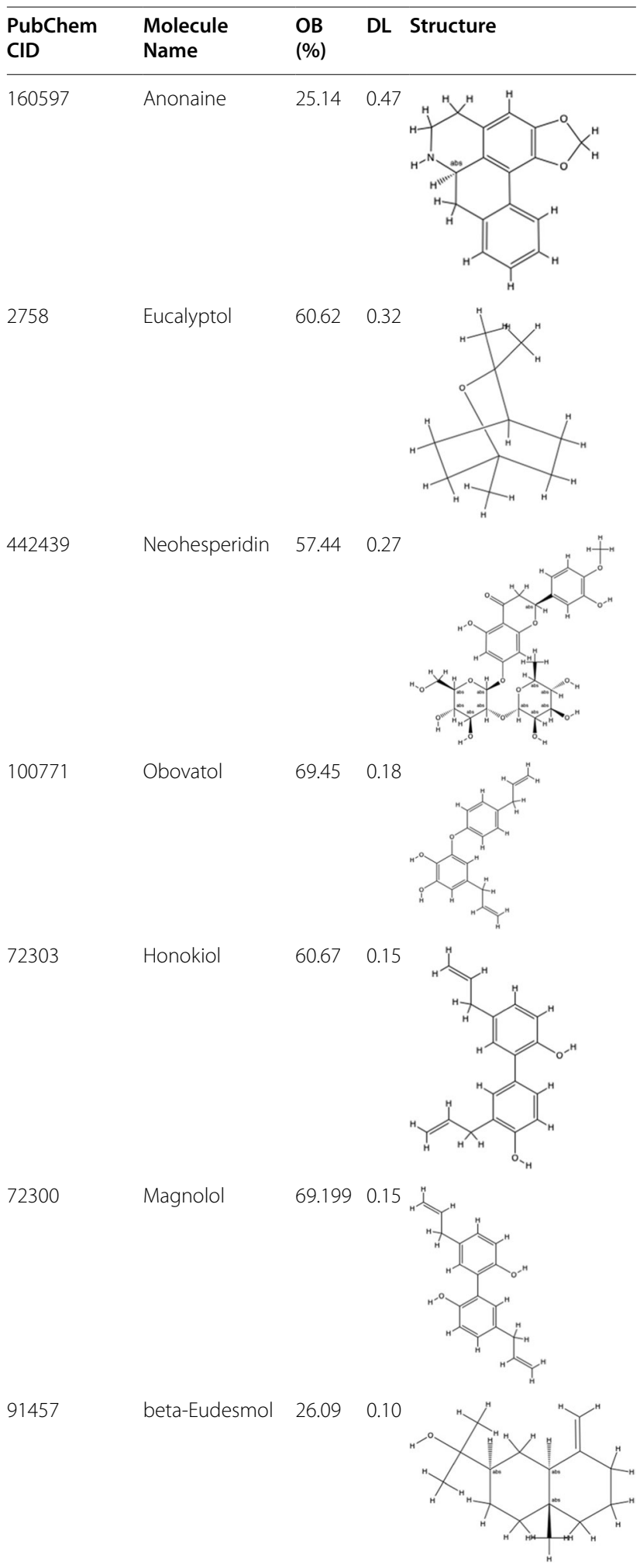

Supplementary Table S8). These signaling pathways also play important role in the pathogenesis of sarcopenia, further indicate that $\mathrm{MC}$ can be used in the treatment of sarcopenia.

\section{Molecular docking analysis}

In order to verify the above analysis results, we conducted molecular docking for the active components of $\mathrm{MC}$ and sarcopenia-related pathogenic proteins. Firstly, we filtered the core proteins of MC-sarcopenia composite targets according to the characteristics of the network topology, using NetworkAnalyzer plug-in unit of Cytoscape (Fig. 4A). After twice filtering, we obtained four core proteins, including AKT1, EGFR, INS, and PIK3CA (Fig. 4B, Supplementary Table S9). Consistent with the sarcopenia-related pathogenic proteins, these four core proteins are mainly involved in PI3K-Akt signaling pathway (hsa04151) and longevity regulating pathway (hsa04213) (Supplementary Figures S4-S6). In retrospect, we matched the targets of active components in the $\mathrm{MC}$ corresponding to these four core proteins. Then, these active components of MC were selected for molecular docking verification, including Honokiol, Magnolol, and Obovatol (Table 2).

The affinity energy of best mode for Honokiol-AKT1 and Magnolol-AKT1 were $-6.2 \mathrm{kcal} / \mathrm{mol}$ and $-6.7 \mathrm{kcal} /$ mol, respectively (Supplementary Table S10, S11). Hydrogen bonding plays a key role in molecular recognition and biology. The result of Honokiol-AKT1 molecular docking showed that there were six hydrogen bondings formed by lysine residues (LYS-14), glutamicacid residues (GLU-17), tyrosine residues (TYR-18), isoleucine residues (ILE-19), arginine residues (ARG-23), arginine residues (ARG-86) in AKT1 protein with Honokiol crystal structure (Fig. 4C). The molecular docking of Magnolol-AKT1 showed one hydrogen bonding formation between tyrosine residues (TYR-38) in AKT1 protein and Magnolol crystal structure (Fig. 4D).

In the process of docking with EGFR, the affinity energy of best mode for Honokiol-EGFR and MagnololEGFR were $-7.0 \mathrm{kcal} / \mathrm{mol}$ and $-7.4 \mathrm{kcal} / \mathrm{mol}$, respectively (Supplementary Table S12, S13). The molecular docking of Honokiol-EGFR showed two hydrogen bondings formation between tryptophan residues (TRP-386) in EGFR protein and Honokiol crystal structure (Fig. 4E). The result of Magnolol-EGFR molecular docking showed that there were three hydrogen bondings formed by alanine residues (ALA-40), glycine residues (GLY-42), lysine residues (LYS-42) in EGFR protein with Magnolol crystal structure (Fig. 4F).

The affinity energy of best mode for Honokiol-INS and Magnolol-INS were $-6.3 \mathrm{kcal} / \mathrm{mol}$ and $-6.0 \mathrm{kcal} / \mathrm{mol}$, respectively (Supplementary Table S14, S15). The result 
A

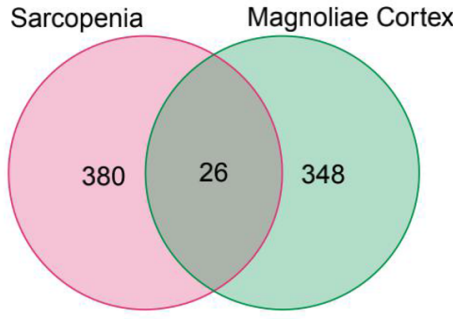

B

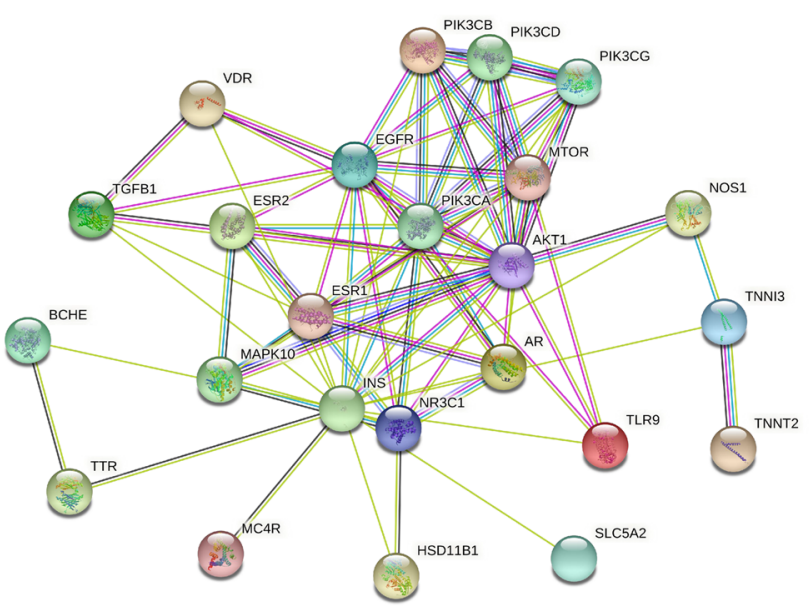

C

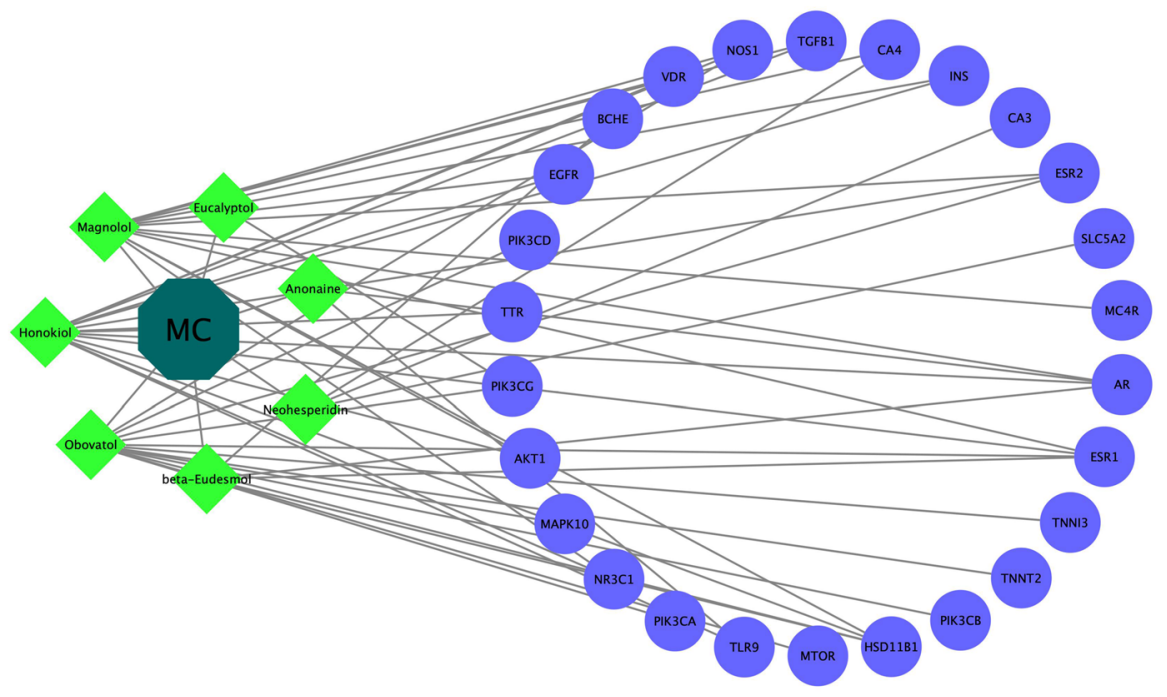

D

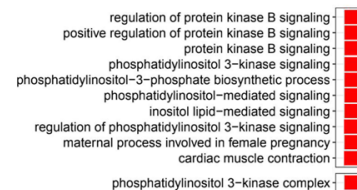

phosphatidylinositol 3 -kinase complex
transferase complex, transterring phosphorus-containing groups

mast cell granule

extrinsic component of membrane
striated muscle thin filament

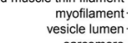

contractile fiber part
myofibril

1-phosphatidylinositol-3-kinase activity
phosphatidylinositol 3 -kinase activity

phosphatidylinositol phosphate kinase activity

phosphatidylinositol kinase activivy
hormone binding

nuclear receptor activivy
nce-specific DNA binding

sequence-specific DNA binding

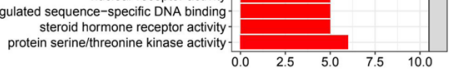

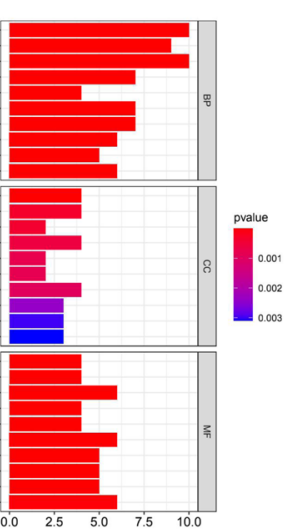

E

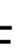

Chemical carcinogenesis - receptor activation

$E$

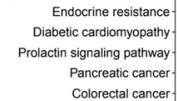

Pancreatic cancer

Colorectal cancer

Relaxin signaling pathway

S signaling pathway

Breast cancer
Phospholipase D signaling pathway

Proteoglycans in cancer

ErbB signaling pathway
PD-L1 expression and PD-1 checkpoint pathway in cancer
Choline metabolism in cancer

Chagas disease

Insulin resistance

HIF-1 signaling pathway

Insulin signaling pattwway

ogen signaling pathway

Autophagy - animal
Gastric cancer

olic fatty liver disease

Type II diabetes mellitus -

Longevity regulating pathway - multiple species.

Central carbon metabolism in cancer.

Longevity regulating pathway.

Progesterone-mediated occyle maturation

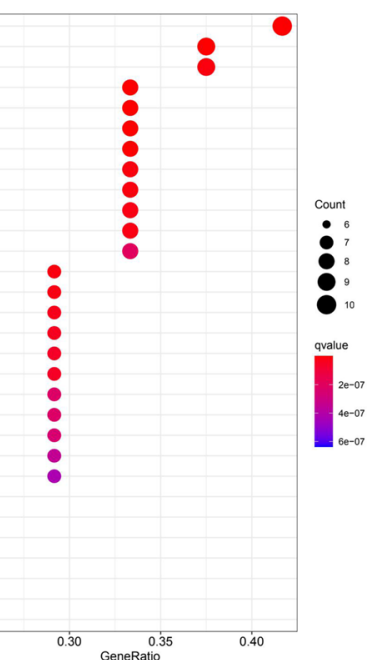

Fig. $3 \mathrm{H}-\mathrm{C}-\mathrm{T}$ network of MC-sarcopenia composite targets. A Venn diagram of the targets in MC and sarcopenia-related pathogenic genes. B PPI network of MC-sarcopenia composite targets. C H-C-T network. D The GO analysis of target proteins involved in sarcopenia treatment by MC. E The KEGG analysis of target protein signal pathway involved in sarcopenia treatment by MC 

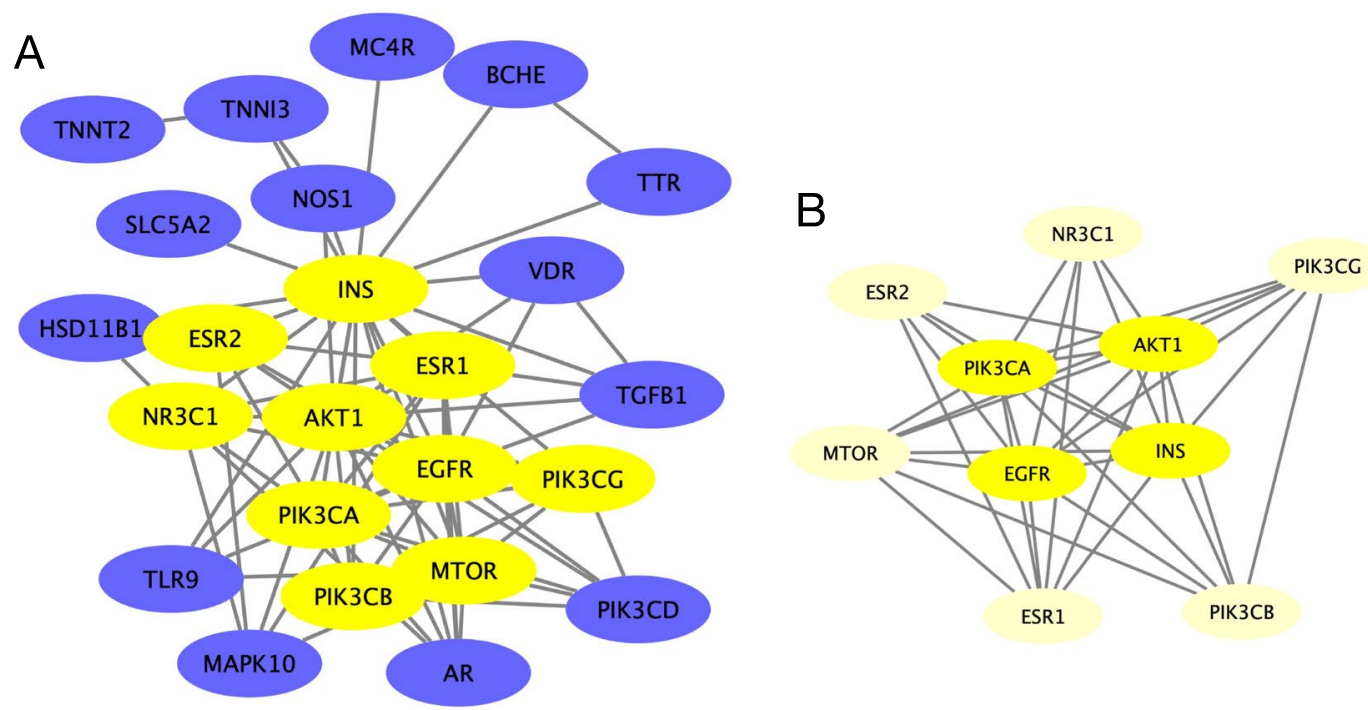

C Honokiol-AKT1

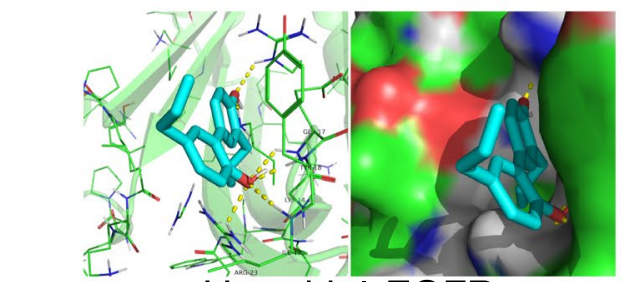

E

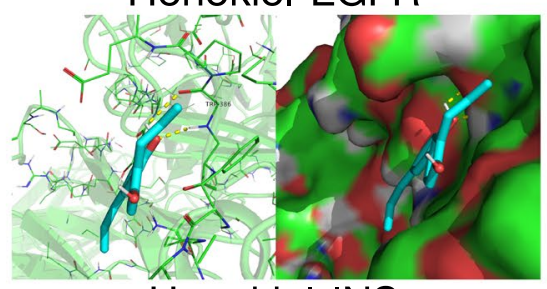

G

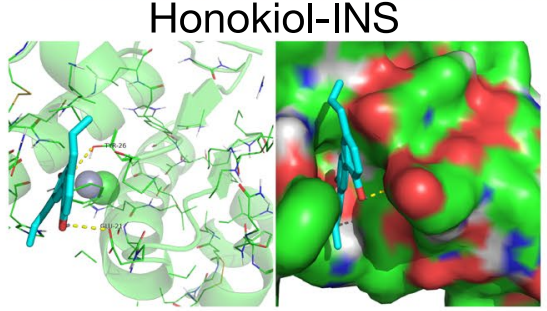

I

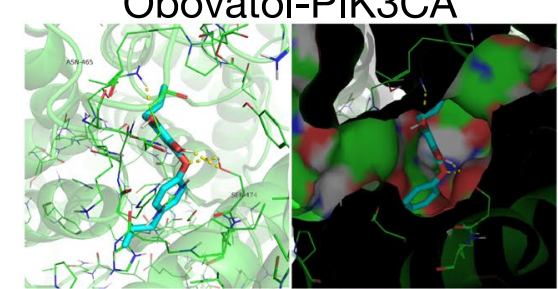

D Magnolol-AKT1

F

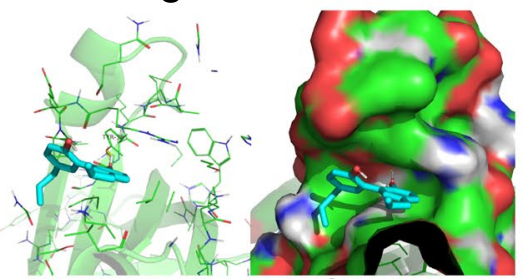

Magnolol-EGFR

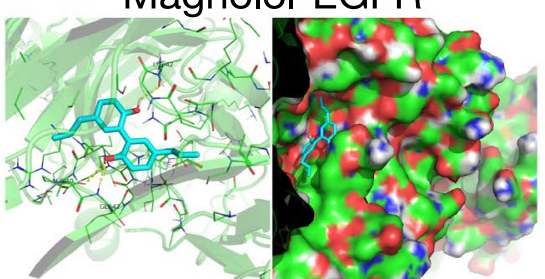

$\mathrm{H}$

Magnolol-INS

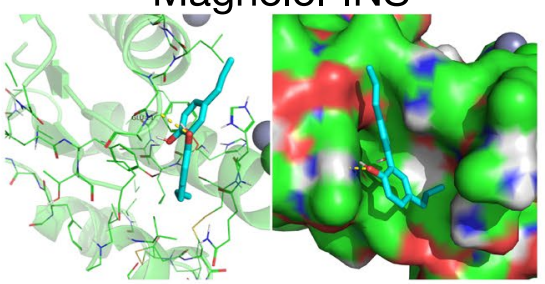

Fig. 4 The protein-ligand of the docking simulation. A-B The process of topological filtering for core proteins of sarcopenia. C Honokiol-AKT1. D Magnolol-AKT1. E Honokiol-EGFR. F Magnolol-EGFR. G Honokiol-INS. H Magnolol-INS. I Obovatol-PIK3CA 
Table 2 MC active components that may have effects on selected core proteins

\begin{tabular}{|c|c|c|c|c|}
\hline Molecule Name & Gene symbol & Protein name & PDB Entry & Protein crystal structure \\
\hline Honokiol, Magnolol & AKT1 & $\begin{array}{l}\text { RAC-alpha serine/ } \\
\text { threonine-protein kinase }\end{array}$ & $1 \mathrm{UNQ}$ & \\
\hline Honokiol, Magnolol & EGFR & Epidermal growth factor receptor & 3POY & \\
\hline Honokiol, Magnolol & INS & Insulin & $1 \mathrm{EV} 3$ & \\
\hline Obovatol & PIK3CA & $\begin{array}{l}\text { Phosphatidylinositol 4,5-bisphosphate 3-kinase } \\
\text { catalytic subunit alpha isoform }\end{array}$ & $5 \mathrm{Fl} 4$ & \\
\hline
\end{tabular}

of Honokiol-INS molecular docking showed that there were two hydrogen bondings formed by glutamicacid residues (GLU-21), tyrosine residues (TYR-26) in INS protein with Honokiol crystal structure (Fig. 4G). The molecular docking of Magnolol-INS showed one hydrogen bonding formation between glutamicacid residues (GLU-21) in INS protein and Magnolol crystal structure (Fig. 4H).

In the process of docking with PIK3CA, the affinity energy of best mode for Obovatol-PIK3CA was-7.4 $\mathrm{kcal} / \mathrm{mol}$ (Supplementary Table S15). The molecular docking of Obovatol-PIK3CA showed three hydrogen bondings formation between asparagine residues (ASN-465), serine residues (SER-474) in PIK3CA protein and Obovatol crystal structure (Fig. 4I).

\section{Discussion}

Optimal intervention for people with sarcopenia is essential because the condition has not only high personal, but also social and economic burdens when untreated [45]. Presence of sarcopenia increases risks for hospitalisation, as well as the cost of care during hospitalisation [46-49]. Recent study showed MC could alleviate muscle wasting in a cisplatin-induced sarcopenia mouse model [9]. However, its effects on sarcopenia patients, as well as potential mechanism, have not been investigated yet. In the current study, results of bioinformatics analysis and network pharmacology analysis showed that main active components of MC target the core proteins of PI3KAkt signaling pathway, EGFR tyrosine kinase inhibitor resistance, longevity regulating pathway, which may play a certain therapeutic role in sarcopenia. Furthermore, the results of molecular docking showed that there exists direct hydrogen bondings between the active components (Honokiol, Magnolol, and Obovatol) of MC and the core proteins of sarcopenia (AKT1, EGFR, INS, and PIK3CA), which verifies our analysis and prediction from another angle. We provided a series of pharmaceutical active ingredients that may be used to treat sarcopenia and speculated their possible mechanisms.

The limited mechanistic understanding of sarcopenia pathophysiology is one of the major reasons why sarcopenia lacks effective treatment measures, thus lack of molecular targets. Previous investigations comparing skeletal muscle in the elderly with that in the young adults have identified mechanisms that drive muscle aging without distinction for the mechanisms that specifically lead to pathological decline and physical disability [50-52]. With the recognition that sarcopenia is a specific pathological disorder no matter in the elderly or the young adults [1], we included three muscle biopsies sequencing data (GSE111006, GSE111010, GSE111016) from GEO database to analyse the difference expressed mRNAs between sarcopenia and non-sarcopenia in elderly patients. After integrating the above sequencing data results with the confirmed candidate genes of sarcopenia in GeneCard, OMIM, Pharmgkb, and DisGeNET databases, we obtained sarcopenia related pathogenic genes (Supplementary Table S2). The sarcopenia related pathogenic genes of our bioinformatics analysis result 
contains many differentially expressed genes detected by RT-PCR method in the study of Patel et al. [53]. According to the results of GO and KEGG enrichment analyses, sarcopenia related pathogenic gene products primarily involve in aging and inflammation related signal pathways, such as longevity regulating pathway (hsa04211), cellular senescence (hsa04218), TNF signaling pathway (hsa04668), IL-17 signaling pathway (hsa04657), EGFR tyrosine kinase inhibitor resistance (hsa01521), PI3KAkt signaling pathway (hsa04151), and endocrine resistance (hsa01522) et al.. These sarcopenia related signaling pathways were similar to those explored in recent years. Wilson et al. considered that age-related decline in immune cell function, increased inflammation and the dysregulation of the PI3K-Akt pathway in neutrophils could contribute pathogenically to sarcopenia [54]. Furthermore, inflammaging, characterized by increased levels of proinflammatory cytokines and a reduced level of anti-inflammatory cytokines, contributes to the creation and maintenance of sarcopenia states [55]. In addition, a recent preclinical study has shown that intervention against inflammatory response could effectively alleviate the symptoms of sarcopenia [9]. The drug used in this study was traditional herbal medicine MC of TCM. We thus wonder whether this herbal medicine can be used in the treatment of sarcopenia patients.

Through the method of network pharmacology, we obtained the active components and potential intervention targets of MC. By matching the drug targets of MC with sarcopenia related pathogenic proteins, we obtained the related proteins of $\mathrm{MC}$ involved in sarcopenia intervention, namely $\mathrm{MC}$-sarcopenia targets. After GO and KEGG enrichment analyses performed for these MCsarcopenia targets, we found that proteins affected by $\mathrm{MC}$ active components participate in a large number of key sarcopenia related pathogenic signaling pathways, such as endocrine resistance (hsa01522), PI3KAkt signaling pathway (hsa04151), EGFR tyrosine kinase inhibitor resistance (hsa01521), longevity regulating pathway(hsa04211), etc. (Fig. 3E, Supplementary Table S7). These results suggest that MC is likely to be a promising therapeutic drug for sarcopenia. Then, MC-sarcopenia targets were filtered to obtain four core proteins, namely PIK3CA, AKT1, EGFR, and INS. As PIK3CA and AKT1 are the core components of PI3K-Akt signaling pathway, we speculate that the mechanism of MC participating in sarcopenia treatment may be through the regulation of PI3K-Akt signaling pathway, which also play crucial role in inflammaging [54]. Finally, we used molecular docking technology to verify whether the active components in $\mathrm{MC}$ can interact with sarcopenia related core proteins. As is shown in Fig. 4, there exists at least one hydrogen bonding between residues of sarcopenia related core proteins and MC active components. Surprisingly, there were six hydrogen bondings formed by residues in AKT1 crystal structure with Honokiol (Fig. 4C). Therefore, we speculate the therapeutic effect of $\mathrm{MC}$ on sarcopenia may play a role in the physiological function of AKT1 through Honokiol. However, this needs further research, as well as verification.

A major limitation of the current study is that our results are based on existing databases. Thus, our findings need further validation in cell, animal experiments, and clinical trials, ultimately. First, we need to conduct cellular (in vitro) and animal (in vivo) experiments to verify whether MC has the effects of preventing and treating sarcopenia. Subsequently, it can be grouped according to different $\mathrm{MC}$ active components to filter the active components with better anti-sarcopenia effect, so as to clarify the exact active monomer component or component combinations of anti-sarcopenia in MC. In future research, we should also clarify the following issues: cellular and molecular mechanisms of MC active components in the treatment of sarcopenia, optimal dose of $\mathrm{MC}$ active components for inducing remission with low toxicity, and whether MC is suitable for long-term maintenance treatment of sarcopenia. We hope that we could finally find a monomer component or combination with exact anti-sarcopenia effect and clarify its potential action mechanism, which can be applied to clinic practice and alleviate the current situation of lack of anti-sarcopenia drugs.

\section{Conclusions}

MC might be a promising therapeutic drug for sarcopenia. MC contains potential anti-sarcopenia active compounds. These compounds play a role by regulating the proteins implicated in regulating aging and inflammation related signaling pathways, which are crucial in pathogenesis of sarcopenia. The molecular mechanism underlying the effect of $\mathrm{MC}$ on inducing sarcopenia remission was predicted using a network pharmacology method, thereby providing a theoretical basis for further study of the effective components and mechanism of $\mathrm{MC}$ in the treatment of sarcopenia.

\footnotetext{
Abbreviations

MC: Magnoliae Cortex; GEO: Gene Expression Omnibus; OMIM: Online Mendelian Inheritance in Man; TTD: Therapeutic Target Database; GO: Gene Ontology; KEGG: Kyoto Encyclopedia of Genes and Genomes; BP: Biological process; CC: Cellular composition; MF: Molecular function; TCM: Traditional Chinese Medicine; TCMSP: Traditional Chinese Medicine Systems Pharmacology Database; TCMID: Traditional Chinese Medicine Information Database; BATMAN-TCM: Bioinformatics analysis tool for the molecular mechanism of traditional Chinese medicine; ADME: Absorption, distribution, metabolism, and excretion; OB: Oral bio-availability; DL: Drug-likeness; PPI: Protein-protein interaction; H-C-T network: "Herbs-Components-Targets" network; DC: Degree Centrality; CC: Closeness Centrality; BC: Betweenness Centrality.
} 


\section{Supplementary Information}

The online version contains supplementary material available at https://doi. org/10.1186/s12863-022-01029-x.

Additional file 1: Table S1. Differentially expressed genes related to sarcopenia in old people of GEO series.Table S2. Integrated data of sarcopenia related pathogenic genes. Table S3. Result of GO enrichment analysis for sarcopenia related pathogenic gene products. Table S4. Result of KEGG enrichment analysis for sarcopenia related pathogenic gene products. Table S5. Result of targets prediction of MC. Table S6. Composite targets of MC and sarcopenia. Table S7. Result of GO enrichment analysis for composite targets of MC and sarcopenia. Table S8. Result of KEGG enrichment analysis for composite targets of MC and sarcopenia. Table S9. Core proteins of MC-sarcopenia composite targets. Table S10. The affinity energy of Honokiol-AKT1. Table S11. The affinity energy of Magnolol-AKT1. Table S12. The affinity energy of Honokiol-EGFR. Table S13. The affinity energy of Magnolol-EGFR. Table S14. The affinity energy of Honokiol-INS. Table S15. The affinity energy of Magnolol-INS. Table S16. The affinity energy of Obovatol-PIK3CA. Figure S1. Sarcopenia related pathogenic gene products involve in EGFR tyrosine kinase inhibitor resistance (hsa01521). Figure S2. Sarcopenia related pathogenic gene products involve in endocrine resistance (hsa01522). Figure S3. Sarcopenia related pathogenic gene products involve in longevity regulating pathway (hsa04211). Figure S4. The GO and KEGG analysis of core sarcopenia-related pathogenic proteins. Figure S5. Core sarcopenia related pathogenic gene products involve in PI3K-Akt signaling pathway (hsa04151). Figure S6. Core sarcopenia related pathogenic gene products involve in longevity regulating pathway (hsa04213).

\section{Acknowledgements}

The authors thank all the participants and instructors who participated in the study.

\section{Authors' contributions}

Xingqi Zhao and Bin Yu conceptualized and designed the study. Xingqi Zhao and Feifei Yuan performed the bioinformatics analysis and network pharmacology analysis. Haoyang Wan and Hanjun Qin performed molecular docking. Xingqi Zhao and Nan Jiang prepared the draft of the manuscript. Feifei Yuan and Bin Yu revised the manuscript. All authors approved the final version of the manuscript.

\section{Funding}

This work was supported by the National Natural Science Foundation of China [grant no. 81802182], the President Foundation of Nanfang Hospital, Southern Medical University [grant no. 2020C027], and the Postdoctoral Science Foundation of China [grant no. 2021M701635].

\section{Availability of data and materials}

All the data can be obtained from the open-source website we provide, and the conclusion can be drawn through the analysis of the relevant software. The datasets generated and/or analysed during the current study are available in the Gene Expression Omnibus (GEO) database repository (GSE1 11006, https://www.ncbi.n/m.nih.gov/geo/query/acc.cgi?acc=GSE111006; GSE111010, https://www.ncbi.nlm.nih.gov/geo/query/acc.cgi?acc=GSE1 1 1010; GSE1 11016, https://www.ncbi.nlm.nih.gov/geo/query/acc.cgi?acc= GSE1 11016), which were used to analyse the differentially expressed genes in muscle tissue.

\section{Declarations}

\section{Ethics approval and consent to participate}

The data of GSE111016 was extracted from a Singapore sarcopenia study, which was approved by the National Healthcare Group Domain-Specific Research Board (NHG DSRB)(reference number 2014/01304), and each participant gave written informed consent. The data of GSE111006 was extracted from a Hertfordshire sarcopenia study, which was approved by the Hertfordshire Research Ethics Committee approval number 07/Q0204/68), and each participant gave written informed consent. The data of GSE111010 was extracted from a Jamaica sarcopenia study, which was approved by the University of West Indies Research Ethics Committee (approval number 180,10/11), and each participant gave written informed consent. This current study is a network integration analysis based on published data. The human related data we used, such as muscle tissue sequencing results, were carefully reviewed and in accordance with the Declaration of Helsinki. This data analysis study protocol has been approved by the ethics board of Nanfang Hospital, Southern Medical University.

\section{Consent for publication}

Not applicable.

\section{Competing interests}

The authors declare that there is no conflict of interest regarding the publication of this paper.

\section{Author details}

${ }^{1}$ Division of Orthopaedics and Traumatology, Department of Orthopaedics \& Guangdong Provincial Key Laboratory of Bone and Cartilage Regenerative Medicine, Nanfang Hospital, Southern Medical University, Guangzhou 510515, China. ${ }^{2}$ Department of Pediatrics, The Third Affiliated Hospital of Guangzhou Medical University, Guangzhou 510150, China.

Received: 7 September 2021 Accepted: 27 January 2022

Published online: 17 February 2022

\section{References}

1. Cruz-Jentoft AJ, Bahat G, Bauer J, Boirie Y, Bruyere O, Cederholm T, et al. Sarcopenia: revised European consensus on definition and diagnosis. Age Ageing. 2019;48:16-31. https://doi.org/10.1093/ageing/afy169.

2. Bischoff-Ferrari HA, Orav JE, Kanis JA, Rizzoli R, Schlogl M, Staehelin HB, et al. Comparative performance of current definitions of sarcopenia against the prospective incidence of falls among community-dwelling seniors age 65 and older. Osteoporos Int. 2015;26:2793-802. https://doi. org/10.1007/s00198-015-3194-y.

3. Schaap LA, van Schoor NM, Lips P, Visser M. Associations of Sarcopenia Definitions, and Their Components, With the Incidence of Recurrent Falling and Fractures: The Longitudinal Aging Study Amsterdam. J Gerontol A Biol Sci Med Sci. 2018;73:1 199-204. https://doi.org/10.1093/gerona/ gl×245.

4. Morley JE, Abbatecola AM, Argiles JM, Baracos V, Bauer J, Bhasin S, et al. Sarcopenia with limited mobility: an international consensus. J Am Med Dir Assoc. 2011;12:403-9. https://doi.org/10.1016/j.jamda.2011.04.014.

5. De Buyser SL, Petrovic M, Taes YE, Toye KR, Kaufman JM, Lapauw B, et al. Validation of the FNIH sarcopenia criteria and SOF frailty index as predictors of long-term mortality in ambulatory older men. Age Ageing. 2016;45:602-8. https://doi.org/10.1093/ageing/afw071.

6. Mueller TC, Bachmann J, Prokopchuk O, Friess H, Martignoni ME. Molecular pathways leading to loss of skeletal muscle mass in cancer cachexiacan findings from animal models be translated to humans? BMC Cancer. 2016;16:75. https://doi.org/10.1186/s12885-016-2121-8.

7. Franceschi C, Campisi J. Chronic inflammation (inflammaging) and its potential contribution to age-associated diseases. J Gerontol A Biol Sci Med Sci. 2014;69(Suppl 1):S4-9. https://doi.org/10.1093/gerona/glu057.

8. Thoma A, Lightfoot AP. NF-kB and Inflammatory Cytokine Signalling: Role in Skeletal Muscle Atrophy. Adv Exp Med Biol. 2018;1088:267-79. https:// doi.org/10.1007/978-981-13-1435-3_12.

9. Hong, M., Han, I. H., Choi, I., Cha, N., Kim, W., Kim, S. K. et al. Magnoliae Cortex Alleviates Muscle Wasting by Modulating M2 Macrophages in a Cisplatin-Induced Sarcopenia Mouse Model. Int J Mol Sci. 2021;22, doi:https://doi.org/10.3390/ijms22063188.

10. Hao E-W, Su Z-X, Gong Y-L, Du Z-C, Yang X, Huang C-T, et al. Analysis on application law of dampness-removing traditional Chinese medicines in treatment of coronavirus disease 2019. Chinese Herbal Medicines. 2021;13:518-24. https://doi.org/10.1016/j.chmed.2021.09.011.

11. Han H, Jung JK, Han SB, Nam SY, Oh KW, Hong JT. Anxiolytic-like effects of 4-O-methylhonokiol isolated from Magnolia officinalis through enhancement of GABAergic transmission and chloride influx. J Med Food. 2011;14:724-31. https://doi.org/10.1089/jmf.2010.1111. 
12. Youn UJ, Fatima N, Chen QC, Chae S, Hung TM, Min B-S. Apoptosisinducing and Antitumor Activity of Neolignans Isolated fromMagnolia officinalisin HeLa Cancer Cells. Phytother Res. 2013;27:1419-22. https:// doi.org/10.1002/ptr.4893.

13. Liu T, Pan Y, Lai R. New mechanism of magnolol and honokiol from Magnolia officinalis against Staphylococcus aureus. Nat Prod Commun. 2014;9:1307-9.

14. Xie NA, Hu C, Guo A, Liang H, Du P, Yin G. Metabolic regulation of magnoIol on the nuclear receptor, liver X receptor. Exp Ther Med. 2015;9:182730. https://doi.org/10.3892/etm.2015.2300.

15. Colinge J, Rix U, Bennett KL, Superti-Furga G. Systems biology analysis of protein-drug interactions. PROTEOMICS - Clinical Applications. 2012;6:102-16. https://doi.org/10.1002/prca.201100077.

16. Zhao J, Jiang P, Zhang W. Molecular networks for the study of TCM pharmacology. Brief Bioinform. 2010;11:417-30. https://doi.org/10.1093/bib/ bbp063.

17. Hopkins AL. Network pharmacology: the next paradigm in drug discovery. Nat Chem Biol. 2008:4:682-90. https://doi.org/10.1038/nchembio.118.

18. Yang, J., Tian, S., Zhao, J. \& Zhang, W. Exploring the mechanism of TCM formulae in the treatment of different types of coronary heart disease by network pharmacology and machining learning. Pharmacological Research. 2020; 159, doi:https://doi.org/10.1016/.jphrs.2020.105034.

19. Wang KX, Gao Y, Lu C, Li Y, Zhou BY, Qin XM, et al. Uncovering the Complexity Mechanism of Different Formulas Treatment for Rheumatoid Arthritis Based on a Novel Network Pharmacology Model. Front Pharmacol. 2020;11:1035. https://doi.org/10.3389/fphar.2020.01035.

20. Qiu T, Wu D, Yang L, Ye H, Wang Q, Cao Z, et al. Exploring the Mechanism of Flavonoids Through Systematic Bioinformatics Analysis. Front Pharmacol. 2018;9:918. https://doi.org/10.3389/fphar.2018.00918.

21. Chen, Z., Wang, X., Li, Y., Wang, Y., Tang, K., Wu, D. et al. Comparative Network Pharmacology Analysis of Classical TCM Prescriptions for Chronic Liver Disease. Frontiers in Pharmacology. 2019; 10, doi:https://doi.org/10. 3389/fphar.2019.01353.

22. Li P, Chen J, Zhang W, Li H, Wang W, Chen J. Network pharmacology based investigation of the effects of herbal ingredients on the immune dysfunction in heart disease. Pharmacol Res. 2019;141:104-13. https:// doi.org/10.1016/.jphrs.2018.12.016.

23. LiW, Mao X, Wu H, Guo M, Su X, Lu J, et al. Deciphering the chemical profile and pharmacological mechanisms of Baihu-Guizhi decoction using ultra-fast liquid chromatography-quadrupole-time-of-flight tandem mass spectrometry coupled with network pharmacology-based investigation. Phytomedicine. 2020;67:153156. https://doi.org/10.1016/.phymed.2019.153156.

24. Li YH, Yu CY, Li XX, Zhang P, Tang J, Yang Q, et al. Therapeutic target database update 2018: enriched resource for facilitating bench-to-clinic research of targeted therapeutics. Nucleic Acids Res. 2018;46:D1121-7. https://doi.org/10.1093/nar/gkx1076.

25 Wishart DS, Feunang YD, Guo AC, Lo EJ, Marcu A, Grant JR, et al. DrugBank 5.0: a major update to the DrugBank database for 2018. Nucleic Acids Res. 2018:46:D1074-82. https://doi.org/10.1093/nar/gkx1037.

26. Pinero J, Bravo A, Queralt-Rosinach N, Gutierrez-Sacristan A, Deu-Pons J, Centeno E, et al. DisGeNET: a comprehensive platform integrating information on human disease-associated genes and variants. Nucleic Acids Res. 2017:45:D833-9. https://doi.org/10.1093/nar/gkw943.

27. Yu G, Wang LG, Han Y, He QY. clusterProfiler: an R package for comparing biological themes among gene clusters. OMICS. 2012;16:284-7. https:// doi.org/10.1089/omi.2011.0118.

28. Kanehisa M, Furumichi M, Sato Y, Ishiguro-Watanabe M, Tanabe M. KEGG: integrating viruses and cellular organisms. Nucleic Acids Res. 2021:49:D545-51. https://doi.org/10.1093/nar/gkaa970.

29. Ru J, Li P, Wang J, Zhou W, Li B, Huang C, et al. TCMSP: a database of systems pharmacology for drug discovery from herbal medicines. J Cheminform. 2014;6:13. https://doi.org/10.1186/1758-2946-6-13.

30. Liu Z, Guo F, Wang Y, Li C, Zhang X, Li H, et al. BATMAN-TCM: a Bioinformatics Analysis Tool for Molecular mechANism of Traditional Chinese Medicine. Sci Rep. 2016;6:21146. https://doi.org/10.1038/srep21146.

31 Huang L, Xie D, Yu Y, Liu H, Shi Y, Shi T, et al. TCMID 2.0: a comprehensive resource for TCM. Nucleic Acids Res. 2018;46:D1117-20. https://doi.org/ 10.1093/nar/gkx1028.

32. Xu X, Zhang W, Huang C, Li Y, Yu H, Wang Y, et al. A novel chemometric method for the prediction of human oral bioavailability. Int J Mol Sci. 2012;13:6964-82. https://doi.org/10.3390/ijms13066964.
33. Acharya B, Chaijaroenkul W, Na-Bangchang K. Therapeutic potential and pharmacological activities of beta-eudesmol. Chem Biol Drug Des. 2021:97:984-96. https://doi.org/10.1111/cbdd.13823.

34. Gfeller D, Michielin O, Zoete V. Shaping the interaction landscape of bioactive molecules. Bioinformatics. 2013;29:3073-9. https://doi.org/10. 1093/bioinformatics/btt540.

35. Szklarczyk D, Morris JH, Cook H, Kuhn M, Wyder S, Simonovic M, et al. The STRING database in 2017: quality-controlled protein-protein association networks, made broadly accessible. Nucleic Acids Res. 2017;45:D362-8. https://doi.org/10.1093/nar/gkw937.

36. Shannon P, Markiel A, Ozier O, Baliga NS, Wang JT, Ramage D, et al. Cytoscape: a software environment for integrated models of biomolecular interaction networks. Genome Res. 2003;13:2498-504. https://doi.org/ 10.1101/gr.1239303.

37. Missiuro PV, Liu K, Zou L, Ross BC, Zhao G, Liu JS, et al. Information flow analysis of interactome networks. PLoS Comput Biol. 2009;5:e1000350. https://doi.org/10.1371/journal.pcbi.1000350.

38. Raman K, Damaraju N, Joshi GK. The organisational structure of protein networks: revisiting the centrality-lethality hypothesis. Syst Synth Biol. 2014:8:73-81. https://doi.org/10.1007/s11693-013-9123-5.

39. Tang Y, Li M, Wang J, Pan Y, Wu FX. CytoNCA: a cytoscape plugin for centrality analysis and evaluation of protein interaction networks. Biosystems. 2015;127:67-72. https://doi.org/10.1016/j.biosystems.2014.11.005.

40. Song W, Ni S, Fu Y, Wang Y. Uncovering the mechanism of Maxing Ganshi Decoction on asthma from a systematic perspective: A network pharmacology study. Sci Rep. 2018;8:17362. https://doi.org/10.1038/ s41598-018-35791-9.

41. Wan Y, Xu L, Liu Z, Yang M, Jiang X, Zhang Q, et al. Utilising network pharmacology to explore the underlying mechanism of Wumei Pill in treating pancreatic neoplasms. BMC Complement Altern Med. 2019;19:158. https://doi.org/10.1186/s12906-019-2580-y.

42. Xiong $Y$, Yang Y, Xiong W, Yao Y, Wu H, Zhang M. Network pharmacologybased research on the active component and mechanism of the antihepatoma effect of Rubia cordifolia L. J Cell Biochem. 2019;120:12461-72. https://doi.org/10.1002/jcb.28513.

43. Li J, Ma X, Liu C, Li H, Zhuang J, Gao C, et al. Exploring the Mechanism of Danshen against Myelofibrosis by Network Pharmacology and Molecular Docking. Evid Based Complement Alternat Med. 2018;2018:8363295. https://doi.org/10.1155/2018/8363295.

44. Trott O, Olson AJ. AutoDock Vina: improving the speed and accuracy of docking with a new scoring function, efficient optimization, and multithreading. J Comput Chem. 2010;31:455-61. https://doi.org/10.1002/jcc. 21334.

45. Mijnarends DM, Luiking YC, Halfens RJG, Evers S, Lenaerts ELA, Verlaan S, et al. Muscle, Health and Costs: A Glance at their Relationship. J Nutr Health Aging. 2018;22:766-73. https://doi.org/10.1007/ s12603-018-1058-9.

46. Cawthon PM, Lui LY, Taylor BC, McCulloch CE, Cauley JA, Lapidus J, et al. Clinical Definitions of Sarcopenia and Risk of Hospitalization in Community-Dwelling Older Men: The Osteoporotic Fractures in Men Study. J Gerontol A Biol Sci Med Sci. 2017;72:1383-9. https://doi.org/10. 1093/gerona/glw327.

47. Antunes AC, Araujo DA, Verissimo MT, Amaral TF. Sarcopenia and hospitalisation costs in older adults: a cross-sectional study. Nutr Diet. 2017:74:46-50. https://doi.org/10.1111/1747-0080.12287.

48. Steff M, Sima J, Shiells K, Holmerova I. The increase in health care costs associated with muscle weakness in older people without long-term illnesses in the Czech Republic: results from the Survey of Health, Ageing and Retirement in Europe (SHARE). Clin Interv Aging. 2017;12:2003-7. https://doi.org/10.2147/CIA.S150826.

49. Sousa AS, Guerra RS, Fonseca I, Pichel F, Ferreira S, Amaral TF. Financial impact of sarcopenia on hospitalization costs. Eur J Clin Nutr. 2016;70:1046-51. https://doi.org/10.1038/ejcn.2016.73.

50. Drummond MJ, McCarthy JJ, Sinha M, Spratt HM, Volpi E, Esser KA, et al. Aging and microRNA expression in human skeletal muscle: a microarray and bioinformatics analysis. Physiol Genomics. 2011;43:595-603. https:// doi.org/10.1152/physiolgenomics.00148.2010.

51. Phillips BE, Williams JP, Gustafsson T, Bouchard C, Rankinen T, Knudsen S, et al. Molecular networks of human muscle adaptation to exercise and age. PLoS Genet. 2013;9:e1003389. https://doi.org/10.1371/journal.pgen. 1003389. 
52. Rivas DA, Lessard SJ, Rice NP, Lustgarten MS, So K, Goodyear LJ, et al. Diminished skeletal muscle microRNA expression with aging is associated with attenuated muscle plasticity and inhibition of IGF-1 signaling. FASEB J. 2014;28:4133-47. https://doi.org/10.1096/f.14-254490.

53. Patel HP, Al-Shanti N, Davies LC, Barton SJ, Grounds MD, Tellam RL, et al. Lean mass, muscle strength and gene expression in community dwelling older men: findings from the Hertfordshire Sarcopenia Study (HSS). Calcif Tissue Int. 2014;95:308-16. https://doi.org/10.1007/s00223-014-9894-z.

54. Wilson D, Jackson T, Sapey E, Lord JM. Frailty and sarcopenia: The potential role of an aged immune system. Ageing Res Rev. 2017;36:1-10. https://doi.org/10.1016/j.arr.2017.01.006.

55. Sendama W. The effect of ageing on the resolution of inflammation. Ageing Res Rev. 2020;57:101000. https://doi.org/10.1016/j.arr.2019.101000.

\section{Publisher's Note}

Springer Nature remains neutral with regard to jurisdictional claims in published maps and institutional affiliations.

- fast, convenient online submission

- thorough peer review by experienced researchers in your field

- rapid publication on acceptance

- support for research data, including large and complex data types

- gold Open Access which fosters wider collaboration and increased citations

- maximum visibility for your research: over $100 \mathrm{M}$ website views per year

At BMC, research is always in progress.

Learn more biomedcentral.com/submissions 